ORIGINAL ARTICLE

\title{
Cranial computed tomography in trauma: the accuracy of interpretation by staff in the emergency department
}

\author{
B Mucci, C Brett, L S Huntley, M K Greene
}

Emerg Med J 2005;22:538-540. doi: 10.1136/emj.2003.013755

See end of article for authors' affiliations

.......................

Correspondence to Dr B Mucci, Consultant Radiologist, Department of Radiology, West

Cumberland Hospital, Whitehaven, Cumbria CA28 8JG, UK

brian.mucci@

ncumbria-acute.nhs.uk

Accepted for publication 2 July 2004
Objectives: Cranial computed tomography (CT) is replacing skull radiography in head trauma. Rapid radiological opinions on these images may not always be available. We assessed the ability of our permanent emergency department staff to interpret the images.

Methods: A retrospective series of 100 consecutive cases was reviewed and interpreted by five permanent emergency department medical staff, and their interpretation compared with the consensus opinion of two radiologists.

Results: An overall agreement of $86.6 \%$ (95\% confidence interval (Cl) 83.4 to 89.9 ) was achieved, with a false negative rate of $4.2 \%(95 \% \mathrm{Cl} 3.9$ to 4.3$)$. No findings that would have changed the overnight management of any patient were missed.

Conclusions: Our results for CT scans are similar to studies of interpretation of other radiographic images in emergency departments. Our emergency staff could safely make the initial interpretation of cranial CT images in trauma out of hours, and formal reporting may wait until a suitably experienced radiologist is available.
$\mathrm{D}$ screpancies in plain radiograph interpretation between emergency department (ED) staff and radiologists have been the subject of study by various groups. ${ }^{1-6}$ Rates of disagreement in the range of $8-11 \%$ are reported; however, many of the misinterpretations have no clinical impact. Initial interpretation of plain radiographs by ED staff, supported by subsequent radiologist reports, has been standard practice for many years. Differences in the interpretation of computed tomography (CT) head scans between clinicians and neuroradiologists have also been investigated. Mehta et al showed a disagreement between clinicians and neuroradiologists in over $20 \%$ of cases and suggested that management change would be seen in 6.6\%. ${ }^{7}$ Similarly, Alfaro et al found discordance in $38.7 \%$ of cases, in $11.4 \%$ of which the disagreement was major. ${ }^{8}$ This study was not confined to trauma cases, and the timescale in management change was not significant in most cases. They concluded that mismanagement due to clinicians' opinion of the images is rare.

Recent clinical guidelines in the UK from the National Institute of Clinical Excellence ${ }^{9}$ (NICE) and the Scottish Intercollegiate Guidelines Network $^{10}$ (SIGN) promote the widespread use of cranial CT in head injury, with skull radiographs being relegated to a few specialist uses. This will result in a large increase in the number of cranial CT scans performed. The NICE guidelines state that image interpretation should be carried out by a "competent clinician", but who this should be is not stated. In this study we ask whether ED staff could make the initial interpretation of these images in the same way as they do for plain radiographs, or if this would result in serious missed diagnoses, which would alter immediate management.

\section{METHODS}

All out of hours CT scans performed in our hospital are recorded by our computerised radiology management system and a manual paper record kept by the radiographers. These records and the radiology request forms were searched to identify 100 consecutive cases where the patient presented with a known or suspected history of trauma. Cases where there were medical reasons for the examination but no suspicion of trauma were excluded. The study group was a mixed population of adults and children.

The images and original radiologists' reports were reviewed by two experienced consultant radiologists (BM and $\mathrm{LH}$ ). In three cases the original report was equivocal. Using the images, clinical history, and outcomes, a consensus "reference standard" report was reached in the 100 cases. The findings were classified into normal $(n=59)$, abnormal related to presentation $(\mathrm{n}=36)$, and abnormal but incidental finding $(\mathrm{n}=5)$.

Original hard copy laser images were reviewed by five permanent members of the ED medical staff (two consultants, three staff grades). A unique study number identified each case. The images were not anonymised but all were at least a year old and were randomly ordered to minimise the risk of cases being recognised. The images were viewed using our standard head window levels in all cases (level +35 , width 60 Hounsfield units). Where available in the patient's folder, bone window images were also provided. A short history for each case was given, which was transcribed from the original request form. The readers recorded their interpretations of the images using a combination of free text and diagrammatic representation of the scan image. They were asked to indicate in each case if neurosurgical referral was warranted on the basis of the imaging findings.

The ED staff reports were compared with the radiological consensus, and cases classified into true positive, true negative, false positive, and false negative interpretations. For the purpose of false negative classification, missed incidental findings were not included, as they were classified as true negatives.

\section{RESULTS}

The results from the ED readers compared with the radiological standard are given in table 1 .

Abbreviations: $\mathrm{CT}$, computed tomography; $\mathrm{ED}$, emergency department; NICE, National Institute of Clinical Excellence; SIGN, Scottish Intercollegiate Guidelines Network 
Table 1 Results from the ED readers compared with the radiological standard

\begin{tabular}{lllll}
\hline Reader & $\begin{array}{l}\text { True } \\
\text { negative }\end{array}$ & $\begin{array}{l}\text { True } \\
\text { positive }\end{array}$ & $\begin{array}{l}\text { False } \\
\text { negative }\end{array}$ & $\begin{array}{l}\text { False } \\
\text { positive }\end{array}$ \\
\hline A & 49 & 35 & 4 & 12 \\
B & 53 & 33 & 4 & 10 \\
C & 54 & 32 & 5 & 9 \\
D & 57 & 34 & 5 & 4 \\
E & 54 & 32 & 5 & 9 \\
\hline
\end{tabular}

The false negative rates ranged from 4 to $5 \%$, with a mean of $4.2 \%$ (95\% confidence interval (CI) 3.9 to $5.3 \%$ ). Mean agreement with the gold standard is $86.6 \%$ (95\% confidence interval 83.4 to $89.9 \%$ ). Review of the false negative responses showed that seven cases accounted for all 23 false negative readings (table 2 ).

No case was a false negative for all five readers. Radiologically, all the false negative cases had subtle findings, and none was considered to merit immediate transfer to the regional neurosurgical unit under NICE guidelines. ${ }^{9}$

Review of the false positive responses showed a wider spread of cases. In total, 43 false positive reads were spread across 25 different scan areas. Most were imaging artefacts or normal anatomical variants. A large but normal cisterna magna was identified by four readers as a possible abnormality needing a surgical opinion. Various streak artefacts were called bleeding in three cases (six reading episodes).

No abnormality warranting immediate neurosurgical referral was missed by the clinicians in the study.

\section{DISCUSSION}

Cranial CT is likely to replace skull radiography for patients with significant head injury in the near future. Initial interpretation of these images by competent clinicians will be required. If ED staff could do this, it is likely to be the most rapid and cost effective process, but it must be shown to be safe.

Overall accuracy of ED staff in the initial interpretation of radiographs has been studied in previous papers. ${ }^{1-5}$ False negative rates around $1-3 \%$ of examinations are common, and as high as $11 \%$ are seen. Vincent $e t a l^{6}$ assessed junior ED staff on a set of purely abnormal films and found an error rate of 35\%. Most missed diagnoses do not adversely affect clinical outcome in the period between initial interpretation and radiological review. ${ }^{41}$

We assessed the accuracy of ED staff in the interpretation of cranial CT images in the trauma setting. The overall accuracy of $86.6 \%$ compares with results in plain film interpretation, as does the false negative rate of $4.2 \%$. Abnormalities in post-trauma CT scans are more likely to be life threatening than other radiographic findings from the

\begin{tabular}{|c|c|c|}
\hline $\begin{array}{l}\text { False } \\
\text { negative } \\
\text { case }\end{array}$ & $\begin{array}{l}\text { Misread by } \\
\text { readers ( } \mathrm{n})\end{array}$ & Finding not detected \\
\hline$A$ & 1 & Closed depressed fracture \\
\hline B & 4 & Small amount of intracranial air \\
\hline C & 4 & Blood in sylvian fissure \\
\hline $\mathrm{D}$ & 4 & Subarachnoid haemorrhage at tentorium \\
\hline $\mathrm{E}$ & 4 & Subarachnoid frontal haemorrhage \\
\hline $\mathrm{F}$ & 4 & Small amount of intracranial air \\
\hline G & 2 & Subarachnoid haemorrhage \\
\hline
\end{tabular}

ED, therefore it is important that initial interpretation has a high sensitivity. We found no false negative reads in which a missed diagnosis would have adversely affected patient outcome overnight. Although the guidelines ${ }^{9}{ }^{10}$ state that all new surgically significant abnormalities should be discussed with a neurosurgeon, a number of abnormalities are listed as not being significant, including localised subarachnoid haemorrhage, isolated pneumocephaly, and closed depressed fractures not penetrating the inner table. All of our false negative reads were in this group. Although accuracy and sensitivity were good in our study, there was significant interobserver variation. Robinson et $a l^{12}$ found a significant variation in opinion even when experienced radiologists were studied. Our results were obtained from staff with no formal training in CT interpretation. There is good evidence that accuracy can be improved with suitable training. ${ }^{213}{ }^{14}$ False positive reports leading to unnecessary neurosurgical referral should therefore reduce with training and experience.

The use of "night hawk" radiologists is seen in North America, ${ }^{15}$ but with current radiology manpower in the UK this is impractical for most hospitals. Training departments could use trainee radiologists who are being taught image interpretation and need this experience; however, their performance is variable and may not be better than permanent ED staff. Our results from permanent ED staff are similar to those obtained from studies of radiologists in training. ${ }^{16-18}$ Teleradiology is available and widely used in the UK, but is relatively slow and has not been used for the volume of work needed to meet the recent head injury guidelines. Ours is the smallest district hospital in England, yet we perform over 850 skull radiographs per year, with over $600(70 \%)$ being requested outside normal working hours. Replacing skull radiographs with CT will have a large impact on radiology departments. As subspecialisation amongst radiologists increases, it is becoming more frequent to find that the on call radiologist does not perform CT during his normal work. Teleradiology to neurosurgical centres is generally available to discuss problem cases. Centralised CT reporting centres could be established, but the few currently available have proved too expensive for our hospital to employ them.

It has been longstanding practice for ED staff to make the initial interpretation of radiographs from their department. Imaging findings are only part of the picture in deciding whether trauma patients need neurosurgical referral. The clinical setting is also relevant. The level of staff we studied (consultant and staff grades) are available to our department at all times and we feel that they could safely make the initial interpretation of cranial CT scans in these cases. The key immediate question is: "is there structural intracranial damage?", ${ }^{10}$ and with training and audit, ED staff should be able to answer this. Subsequent review by radiologists will detect some additional abnormalities and clarify the false positive readings. Inevitably, some patients presenting with a history of possible trauma are in fact medical cases such as stroke. While guidelines refer to trauma, it is not possible to compartmentalise trauma cases entirely. Radiological review in conjunction with ongoing audit will be required.

Direct access to cranial CT for trauma or possible trauma is desirable, and our study suggests that ED staff could safely carry out initial interpretation.

\section{Authors' affiliations}

B Mucci, L S Huntley, Department of Radiology, West Cumberland Hospital, Whitehaven, Cumbria CA28 8JG, UK

C Brett, M K Greene, Department of Accident and Emergency, West Cumberland Hospital, Whitehaven, Cumbria CA28 8JG, UK

Competing interests: none declared 


\section{REFERENCES}

1 Guly HR. Diagnostic errors in an accident and emergency department. Emer Med J 2001;18:263-9.

2 Espinosa JA, Nolan TW. Reducing errors made by emergency physicians in interpreting radiographs: longitudinal study. BMJ 2000;320:737-40.

3 De Lacey GJ, Barker A, Harper J. An assessment of the clinical effects of reporting accident and emergency radiographs. Br J Radiol 1980;53:304-8.

4 Wardrope J, Chenells PM. Should all casualty radiographs be reviewed? BMJ 1985;290: 1638-40.

5 Benger JR, Lyburn ID. What is the effect of reporting all emergency department radiographs? Emerg Med J 2003;20:40-3.

6 Vincent CA, Driscoll PA, Audley RJ, et al. Accuracy of detection of radiographic abnormalities by junior doctors. Arch Emerg Med 1988;5:101-9.

7 Mehta A, Mills TD, Jones JO, et al. Accuracy of interpretation of emergency cranial computed tomography scans by radiology residents and the senior attending member of the requesting clinical team. Radiology 2001;221(RSNA abstract supp): 440 .

8 Alfaro D, Levit MA, English DK, et al. Accuracy of interpretation of cranial computed tomography scan in an emergency training program. Ann Emerg Med 1995;25: 169-74.

9 National Institute for Clinical Excellence. Clinical guideline 4: head injury. London: NICE, 2003.

10 Scottish Intercollegiate Guideline Network. Guideline 46: Early management of acute head injury. Edinburgh: SIGN, 2000.
11 Eschempati SR, Flomenbaum N, Seifert C, et al. Alterations of preliminary readings on radiographic examination minimally affect outcomes of trauma patients discharged from the emergency department. J Trauma 2000;48:654-8.

12 Robinson PJA, Wilson D, Coral A, et al. Variation between experienced observers in the interpretation of accident and emergency radiographs. Br J Radiol 1999;72:323-30

13 Levit MA, Dawkins R, Williams V, et al. Abbreviated educational session improves cranial computed tomography scan interpretations by emergency physicians. Ann Emerg Med 1997;30:616-21.

14 Peron AD, Huff JS, Ullrich CG, et al. A multicentre study to improve emergency residents' recognition of intracranial emergencies on computed tomography. Ann Emerg Med 1998;32:554-62.

15 Mann FA, Danz PL. The night stalker effect: quality improvements with a dedicated night call rotation. Invest Radiol 1993;28:92-6.

16 Roszler MH, McCarroll KA, Rashid T, et al. Resident interpretation of emergency computed tomographic scans. Invest Radiol 1991;26:374-6.

17 Wysoski MG, Nassar CJ, Koenigsberg RA, et al. Head trauma: CT scan interpretation by radiology residents versus staff radiologists. Radiology 1998;208:125-8.

18 Hillier JC, Tattersall DJ, Gleeson FV. Trainee reporting of computed tomography examinations: do they make mistakes and does it matter? Clin Radiol 2004;59:159-62.

\section{bmjupdates+}

bmjupdates+ is a unique and free alerting service, designed to keep you up to date with the medical literature that is truly important to your practice.

bmjupdates+ will alert you to important new research and will provide you with the best new evidence concerning important advances in health care, tailored to your medical interests and time demands.

\section{Where does the information come from?}

bmjupdates+ applies an expert critical appraisal filter to over 100 top medical journals A panel of over 2000 physicians find the few 'must read' studies for each area of clinical interest

Sign up to receive your tailored email alerts, searching access and more...

www.bmjupdates.com 